\title{
SOLVABLE LIE GROUPS AND LOCALLY EUCLIDEAN RIEMANN SPACES
}

\section{AUSLANDER AND M. AUSLANDER ${ }^{1}$}

In [4] Mostow remarks that nonabelian solvable groups can operate transitively on the torus. In this paper we characterize the simply connected, connected solvable Lie groups (for the rest of this paper a solvable Lie group will always be understood to be connected and simply connected) which can operate transitively on the torus with a discrete isotropy group. The characterization is contained in the following theorem.

Theorem A. A necessary and sufficient condition for an $n$ dimensional solvable Lie group to act transitively in the $n$ dimensional torus with a discrete isotropy group is that $S$ can be written as

$$
1 \rightarrow R^{s} \rightarrow S \rightarrow R^{t} \rightarrow 1
$$

where $R^{i}$ denotes the $i$ dimensional vector space over the reals and we have the following conditions satisfied:

1. $s+t=n$,

2. The extension is a split extension,

3. The action of $R^{t}$ on $R^{s}$ is a compact group.

Now let $R(n)$ denote the group of rigid motions of euclidean $n$ dimensional space. Then the pure translations, $T$, constitute a normal subgroup of $R(n)$ isomorphic to $R^{n}$, where $R^{n}$ denotes the $n$ dimensional vector space over the reals. Further the orthogonal group $O(n)$ is a subgroup of $R(n)$ such that $O(n) \cap T=1$, where 1 denotes the identity element, and $R(n)=O(n) T$; i.e. $R(n)$ is the split extension of $O(n)$ by $T$. Hence the Lie algebra, $\operatorname{lr}(n)$, of $R(n)$ is, as a vector space the direct sum of the Lie algebra, $l o(n)$, of $O(n)$ and $R^{n}$. Let $p$ denote the projection of $\operatorname{lr}(n)$ onto $R^{n}$. Let $L$ be an $n$-dimensional subalgebra of $\operatorname{lr}(n)$ such that $L$ is mapped onto $R^{n}$ by $p$ and let $S$ be the connected subgroup of $R(n)$ whose Lie algebra is $L$. A Lie group obtained in this fashion will be said to have property I'. If $S$ satisfies property $\mathrm{I}^{\prime}$ and in addition $S$ has a discrete subgroup $\Gamma$ such that $S / \Gamma$ is compact, then we will say that $S$ has property I.

TheOREM B. A necessary and sufficient condition for a solvable Lie

Received by the editors April 30, 1958.

1 This paper was written while both authors were National Science Foundation Postdoctoral Fellows. 
group to act transitively and with a discrete isotropy group on a torus is that $S$ be isomorphic to a group with property I.

Naturally associated with the torus are the class of compact locally euclidean riemann spaces, i.e. riemann spaces with curvature and torsion zero. The fundamental group of such spaces has been algebraically characterized in [1] (for a resume see \$2) and each of these has a torus as covering space. The second main result is the characterization of which locally euclidean riemann spaces are also solvmanifolds (compact homogeneous spaces of solvable Lie groups) with a discrete isotropy group. We will characterize such spaces by group theoretically characterizing the fundamental group of such spaces. Our main result may be formulated as follows:

Theorem C. Necessary and sufficient conditions for a group $\Gamma$ to be the fundamental group of a compact locally euclidean riemann manifold which is also a solvmanifold are that $\Gamma$ have no elements of finite order and that $\Gamma$ can be represented in the exact sequence

$$
1 \rightarrow K \rightarrow \Gamma \rightarrow F \rightarrow 1,
$$

where $K$ and $F$ are free abelian of rank $s$ and $t$ respectively, $s+t=n$, and where the extension has the following further propcrties:

1. The action of $F$ on $K$ is a finite group.

2. The group extension determines an element of finite order in $H^{2}(F, K)$.

3. The action of $F$ on $K \otimes R$ induced by the mapping $k \rightarrow k \otimes 1$ can be extended to an action of $F \otimes R$ on $K \otimes R$, the tensor products being taken over the integers.

Condition 3 in Theorem $\mathrm{C}$ is easily seen to be equivalent to saying that the holonomy group of $\Gamma$ lie on a torus in $G L(n)$ whose dimension is the rank of $F$.

REMARK 1. These results are not related to the study of the group of isometries of compact locally euclidean riemann spaces. It merely amounts to a study of which compact locally euclidean spaces are homogeneous spaces of solvable Lie groups with a discrete isotropy group. We do not require that the group operate on the homogeneous space by isometries. As a matter of fact, it is known that the only compact locally euclidean riemann spaces with a transitive group of isometries is the torus and then the group of isometries is isomorphic to the torus group.

REMARK 2. If $\Gamma$ is a discrete subgroup of the solvable Lie groups $S$ and $S^{*}$ such that $S / \Gamma$ and $S^{*} / \Gamma$ are compact, then $S$ and $S^{*}$ have the same dimension. To see this we observe that $\Gamma$ acts properly discon- 
tinuously on the acyclic spaces $S$ and $S^{*}$ and thus $H^{i}\left(S / \Gamma, I_{2}\right)$ $\approx H^{i}\left(\Gamma, I_{2}\right) \approx H^{i}\left(S^{*} / \Gamma, I_{2}\right)$ for all $i$, where $I_{2}$ denotes the integers $\bmod 2$ and $\Gamma$ operates trivially on $I_{2}$. Since $S / \Gamma$ and $S^{*} / \Gamma$ are compact manifolds, it follows that $S / \Gamma$ and $S^{*} / \Gamma$ have the same dimension. From this it follows that $S$ and $S^{*}$ have the same dimension.

1. On subalgebras of $\operatorname{lr}(n)$. In this section we will show that every solvable subgroup $S$ of $R(n)$ satisfying property I has the property that $S / \Gamma$ is homeomorphic to a compact locally euclidean riemann space. We will also give a necessary and sufficient condition for a group having property $\mathrm{I}^{\prime}$ to have property $\mathrm{I}$.

We can define a point set mapping $p^{*}: R(n) \rightarrow T$ as follows: Every element in $R(n)$ can be written uniquely as $t \phi$, where $\phi \in O(n)$ and $t \in T$. Define $p^{*}: R(n) \rightarrow T$ by $p^{*}(x)=t$.

THEOREM 1.1. Let $S$ be a subgroup of $R(n)$ with property I'. Then $p^{*}$ maps $S$ homeomorphically onto $T$.

Proof. The hypothesis is equivalent to the statement that the Jacobian of the mapping $p^{*}$ is nondegenerate at the origin of $T$. Hence $p^{*}$ is a 1-1 mapping of a neighborhood $U$ of the identity of $S$ onto a neighborhood $U^{*}$ of the origin in T. Hence $U^{*}$ contains a sphere of radius greater than zero. Now for $s \in S$, we have $p^{*}(s U)$ $=s U^{*}$, where $s U$ denotes left multiplication in $S$ and $s U^{*}$ denotes the action of $R(n)$ on $T$ identified with $R^{n}$. Since $s$ is a rigid motion and $U^{*}$ contains a sphere of radius greater than zero, it is easy to see that the image of $S$ in $T$ under $p^{*}$ is both open and closed and hence all of $T$. We can see that $p^{*}$ is a local homeomorphism from the following facts: $p^{*}$ on $U$ is a homeomorphism, $s$ acting on $S$ and on $T$ is a homeomorphism, and $p^{*}(s U)=s(p U)$. Therefore $S$ is a covering space for $T$ under the mapping $p^{*}$. But since $T$ is simply connected, this implies that $p^{*}$ is a global homeomorphism.

Corollary 1.1. Let $S$ have property I. Then if $\Gamma \subset S$ is a discrete subgroup such that $S / \Gamma$ is compact, $\Gamma$ is the fundamental group of a compact locally euclidean riemann space. Further, $S / \Gamma$ and $R^{n} / \Gamma$ are homeomorphic, where $R^{n}$ denotes the $n$-dimensional real vector space.

PRoof. The proof of this corollary follows easily from Theorem 1.1 and the fact that $s p^{*}=p^{*} s$ for $s \in S$.

Theorem 1.2. If $S$ has property $\mathrm{I}^{\prime}$, then $S$ is a two step solvable Lie group whose commutator subgroup consists of pure translations.

Proof. We first notice that $R(n)$ is a subgroup of $G L(n+1, R)$. Hence $S$ is a Lie group homeomorphic to $R^{n}$ which has a matrix 
representation. It is well-known that such groups must be solvable. But then the projection of $S$ into $O(n)$ must be a connected solvable Lie group of $O(n)$. But this implies that the image of $S$ in $O(n)$ must be abelian. Hence the commutator subgroup of $S$ is abelian and contained in $T$.

THEOREM 1.3. Let $S$ have property $\mathrm{I}^{\prime}$. Then a necessary and sufficient condition for $S$ to have property $\mathrm{I}$ is that the image of $S$ in $O(n)$ be compact.

Proof. Let us assume that $S$ has property I. Then $\Gamma$ contains a subgroup $N$ of finite index consisting of $n$ linearly independent translations. This follows from the fact that $\Gamma$ is the fundamental group of a compact locally euclidean riemann space and the theorem of Bieberbach (see [1]). Consider the homomorphism $f: R(n) \rightarrow O(n)$ with kernel $T$. Then since $N \subset T$,

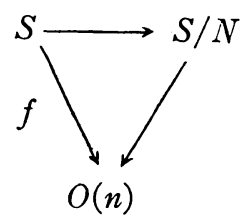

is a commutative diagram. Since $S / N$ is compact, the image of $S$ in $O(n)$ must be compact also. The image of $S$ in $O(n)$ is clearly also abelian, since the kernel, $S \cap T$, contains the commutator subgroup which by Theorem 1.2 consists of pure translations.

Now let us assume that $S / S \cap T$ is compact. Let $(S, S)$ denote the commutator subgroup of $S$. Then $(S, S)$ is contained in $T$ by Theorem 1.2. Hence there is a homomorphism of the vector space $S /(S, S)$ onto a torus group. Hence in the kernel of this homomorphism there is a discrete subgroup $\Gamma_{1}^{*}$ such that $(S /(S, S)) / \Gamma_{1}^{*}$ is compact. Choose $\Gamma_{1}$ in $S \cap T$ which is a preimage of $\Gamma_{1}^{*}$. This is possible since $S \cap T$ is abelian. But $\Gamma_{1}$ consists of pure translations. In $(S, S)$ choose a discrete subgroup $\Gamma_{2}$ such that $(S, S) / \Gamma_{2}$ is compact. Then the group $\Gamma$ generated by $\Gamma_{1}$ and $\Gamma_{2}$ has the property that $\Gamma \subset S$ and $S / \Gamma$ is compact and $\Gamma$ is free abelian on $n$ generators.

REMARK 1. There exist groups which have property I' but not property I.

REMARK 2. In $\S 2$ we will see that the extension

$$
1 \rightarrow S \cap T \rightarrow S \rightarrow S / S \cap T \rightarrow 1
$$

is a split extension.

2. On the groups. Let $\Gamma$ be the fundamental groups of a compact 
locally euclidean riemann manifold. $\Gamma$ is abstractly characterized in [1] by the following two assumptions:

1. $\Gamma$ has a maximal abelian normal subgroup $N$ of finite index which is free abelian on $n$ generators;

2. $\Gamma$ contains no finite subgroups.

Assume now that in addition to being the fundamental group of a compact locally euclidean riemann manifold it is contained as a discrete subgroup of a solvable group $S$ such that $S / \Gamma$ is compact. We will then say that $\Gamma$ has property II.

THEOREM 2.1. Let $\Gamma$ have property II with $\Gamma \subset S$. Then we have the following commutative diagram

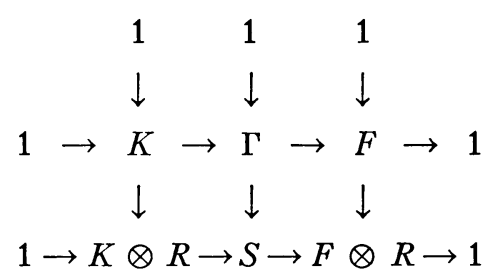

where $K$ and $F$ are finitely generated free abelian groups and $R$ is the real numbers.

Proof. Let $\Gamma \subset S$ and let $M$ be the maximal analytic nilpotent subgroup of $S$. Then $M$ contains the commutator subgroup $(S, S)$ of $S$. Also, since $\Gamma$ has property II, $M \cap \Gamma$ has an abelian subgroup of finite index. Now by [4], we know that, $M / M \cap \Gamma$ is compact. Hence $M$ must be abelian, by [3]. But then $(S, S)$ is abelian and hence the commutator subgroup of $\Gamma$ is abelian and is two step solvable. Let $K=M \cap \Gamma$ and let $F=\Gamma / K$. Since $\Gamma / K=\Gamma / M \cap \Gamma=M \Gamma / M \subset S / M, F$ is isomorphic to a uniform discrete subgroup of the vector group $S / M$ and is therefore a free abelian group. Hence clearly $M=K \otimes R$ and $S / M=F \otimes R$.

Lemma 2.1. Let $A$ be a finitely generated free abelian group and let $A^{\prime}$ be a subgroup of finite index. Let $\sigma$ be an endomorphism of $A$ which is the identity in $A^{\prime}$. Then $\sigma$ is the identity on $A$.

Proof. We may consider $A$ as the integral points of a finitely generated vector space $V$ over the rational numbers. The endomorphism $\sigma$ of $A$ gives rise to an endomorphism of $V$. From the fact that $A^{\prime}$ is of finite index in $A$ it follows that a basis for $A^{\prime}$ also constitutes a basis for $V$. Since $\sigma$ is the identity on $A^{\prime}, \sigma$ is the identity on $V$ and therefore on $A$. 
Levma 2.2. Suppose $1 \rightarrow K \rightarrow \Gamma \rightarrow^{\phi} F \rightarrow 1$ is an extension of the finitely generated free abelian $F$ by the finitely generated free abelian group $K$. If $N$ is a free abelian subgroup of $\Gamma$ of finite index, the operation of $\phi(N)$ on $K$ induced by the extension is trivial and $\phi^{-1}(\phi(N))$ is an abelian subgroup of $\Gamma$.

Proof. Since $1 \rightarrow K \cap N \rightarrow N \rightarrow \phi(N) \rightarrow 1$ is an abelian extension, we have that $n k n^{-1}=k$ for all $n \in N$ and $k \in K$. Thus $\phi(N)$ operates trivially on $K \cap N$. But $K / K \cap N$ is a finite group. Hence $\phi(N)$ operates trivially on $K$. Therefore $K N=\phi^{-1}(\phi(N))$ is an abelian subgroup of $\Gamma$.

THEOREM 2.2. Suppose $1 \rightarrow K \rightarrow \Gamma \rightarrow^{\phi} F \rightarrow 1$ is an extension of the finitely generated free abelian groups $K$ and $F$. Also suppose that the subgroup $F^{\prime}$ of $F$ consisting of all those elements in $F$ which operate trivially on $K$ is of finite index in $F$. Then the following are equivalent:

(a) There exists a normal abelian subgroup $N$ of $\Gamma$ of finite index.

(b) The element in $H^{2}(F, K)$ which corresponds to the extension $1 \rightarrow K \rightarrow \Gamma \rightarrow F \rightarrow 1$ is of finite order.

(c) $\phi^{-1}\left(F^{\prime}\right)$ is a normal abelian subgroup of $\Gamma$ of finite index containing all abelian subgroups of finite index.

Proof. (a) $\Rightarrow(\mathrm{b})$. Since $\Gamma / N$ is a finite group, $K / K \cap N$ is a finite group. Thus, by Lemma $2.2, N^{\prime}=\phi^{-1}(\phi(N))$ is an abelian subgroup of $\Gamma$ and $\phi\left(N^{\prime}\right)=\phi(N)=C$ operates trivially on $K$. Also, since $\Gamma / N$ is a finite group, $F / C$ is a finite group, say of order $n$. Now the extension $1 \rightarrow K \rightarrow N^{\prime} \rightarrow C \rightarrow 1$ splits, since $N^{\prime}$ is abelian and $C$ is free abelian. Thus the element $\gamma$ in $H^{2}(F, K)$ which corresponds to the extension $1 \rightarrow K \rightarrow \Gamma \rightarrow F \rightarrow 1$ is in the kernel of the mapping $r$ which maps $H^{2}(F, K) \rightarrow H^{2}(C, K)$. Since $F / C$ is a finite group of order $n$, we know that there is a transfer homomorphism $t: I I^{2}(C, K) \rightarrow I^{2}(F, K)$ and that $t r$ is multiplication by $n$. Therefore $n \gamma=0$ and hence $\gamma$ is of finite order.

(b) $\Rightarrow(c)$. Suppose $\gamma \in H^{2}(F, K)$ is of finite order. Let $F^{\prime}$ be the subgroup of $F$ which acts trivially on $K$. Then, since $F^{\prime}$ is a finitely generated free abelian group which acts trivially on the free group $K$, we know that $H^{2}\left(F^{\prime}, K\right)$ is a torsion free group. Thus under the restriction map of $H^{2}(F, K) \rightarrow H^{2}\left(F^{\prime}, K\right)$, we have that $\gamma$ goes into zero. Therefore $1 \rightarrow K \rightarrow \phi^{-1}\left(F^{\prime}\right) \rightarrow F^{\prime} \rightarrow 1$ is the split extension. Since $F^{\prime}$ operates trivially on $K$, we know that $\phi^{-1}\left(F^{\prime}\right)$ is the direct sum $K+F^{\prime}$ and thus abelian. The rest of (c) follows from Lemma 2.2.

(c) $\Rightarrow$ (a). Trivial.

If we have a group extension

$$
1 \rightarrow A \stackrel{i}{\rightarrow} B \stackrel{j}{\rightarrow} C \rightarrow 1
$$


we will say that a subset $K$ of $B$ is a system of representatives for $C^{\prime}, C^{\prime}$ a subgroup of $C$, if $j$ maps $K 1-1$ onto $C^{\prime}$.

Lemma 2.3. Let $\Gamma$ have property II with $\Gamma \subset S$. Then we have the following diagram:

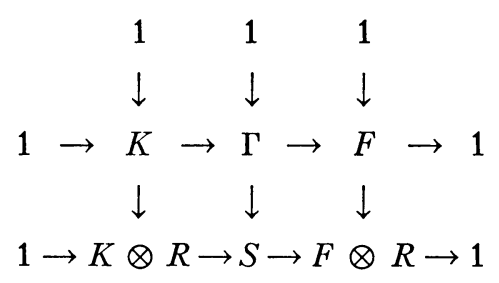

where $K$ is mapped onto $K \otimes 1$ and $F$ is mapped onto $F \otimes 1$. Further there is a subgroup $F^{*}$ of $S$ which is a system of representatives for $F \otimes 1$.

Proof. That the above diagram holds follows from Theorem 2.1. Letting $\Gamma^{\prime}$ be the subgroup $(K \otimes R) \Gamma$ of $S$, we have the commutative diagrams

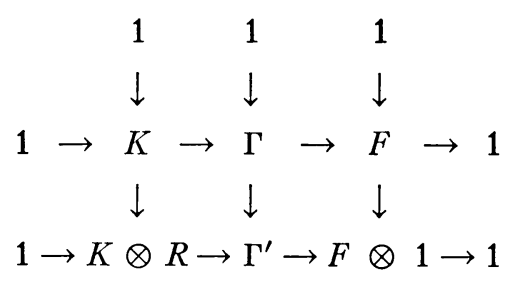

and

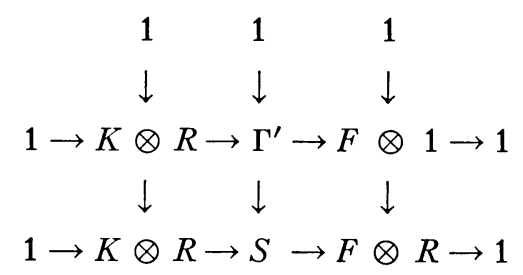

Now let $\gamma$ be the element in $H^{2}(F, K)$ which represents the extension $1 \rightarrow K \rightarrow \Gamma \rightarrow F \rightarrow 1$. Then $\gamma$ is of finite order. It is well known that $H^{2}(F, K \otimes R)$ has no nontrivial torsion subgroups. Consequently under the map $H^{2}(F, K) \rightarrow H^{2}(F, K \otimes R)$ the element $\gamma$ must go into zero. Therefore we deduce from (A) that $1 \rightarrow K \otimes R \rightarrow \Gamma^{\prime} \rightarrow F \otimes 1 \rightarrow 1$ is the split extension. The lemma then follows from diagram (B).

Lemma 2.4. Let $\Gamma$ be a free abelian group and let $\Gamma$ have property II with $\Gamma \subset S$. Then there is a subgroup $\bar{F}$ of $S$ which is in the center of $S$ and a system of representatives for $F \otimes 1$. 
Proof. Since $\Gamma$ is an abelian group, we have that $F$ operates trivially on $K$. Consequently, by standard Lie group theory, it follows that $F \otimes 1$ operates trivially on $K \otimes R$. Let $F^{*}$ be a subgroup of $S$ which is a system of representatives for $F \otimes 1$. Since $F \otimes 1$ operates trivially on $K \otimes R$, then the subgroup of $S$ generated by $F^{*}$ and $K \otimes R$ is abelian.

Now let $s \in S$ and $f \in F^{*}$. Then $s f s^{-1}$ and $f$ have the same image in $F \otimes R$ and consequently $s f s^{-1}=x f$ where $x \in K \otimes R$. Since $K \otimes R$ is a normal subgroup of $S$, we have that $s(K \otimes R) f s^{-1} \subset(K \otimes R) f$ for all $s \in S$. Consequently for a fixed $f \in F^{*}$ we have that each $s \in S$ determines a homeomorphism of $(K \otimes R) f$. Since $(K \otimes R) F^{*}$ is abelian it follows that $(K \otimes R) F^{*}$ operates trivially on $(K \otimes R) f$. Now the factor space $S /(K \otimes R) F^{*}$ is compact. It follows therefore that $S$ operates on $(K \otimes R) f_{0}$ through a compact group.

Since $s k f s^{-1}=s k s^{-1} s f s^{-1}=s k s^{-1} x f$ with $x \in K \otimes R$, we have that the operation of $s$ on $(K \otimes R) f$ is a linear transformation followed by a pure translation. Hence $S$ operates on $(K \otimes R) f$ through a compact group of affine transformations. But a compact group of affine transformations is equivalent to a compact group of rigid motions. Hence there exists a $k(f) \in K \otimes R$ such that $s k(f) f s^{-1}=k(f) f$ for all $s \in S$. Thus $k(f) f$ is in the center of $S$. Now if $f_{1}, \cdots, f_{t}$ are a basis for $F^{*}$, then $k\left(f_{1}\right) f_{1}, \cdots, k\left(f_{t}\right) f_{t}$ generate a group $\bar{F}$ in the center of $S$ which is a system of representatives for $F \otimes 1$.

THEOREM 2.3. Let $\Gamma$ have property II and $\Gamma \subset S$, then $S$ is a split extension.

Proof. Since $\Gamma$ always has a subgroup of finite index (and hence also has property II with respect to $S$ ) which is free abelian it suffices to prove the theorem in the case $\Gamma$ is free abelian. Consider

$$
1 \rightarrow K \otimes R \rightarrow S \rightarrow F \otimes R \rightarrow 1
$$

and let $F^{*}$ be a system of representatives for $F \otimes 1$ which is a subgroup in the center of $S$. Then we may consider the exact sequence

$$
1 \rightarrow K \otimes R \rightarrow S / F^{*} \rightarrow F \otimes R / F \otimes 1 \rightarrow 1 .
$$

But $F \otimes R / F \otimes 1$ is a compact group. Hence we may apply the result of Iwasawa [2, pp. 521, Lemma 3.7] that the extension of a vector group by a compact group always splits. Therefore we have

$$
\begin{aligned}
& 1 \rightarrow K \otimes R \rightarrow S \rightarrow F \otimes R \rightarrow 1 \\
& 1 \rightarrow K \otimes R \rightarrow S / F^{*} \rightarrow F \otimes R / F \otimes 1 \rightarrow 1
\end{aligned}
$$

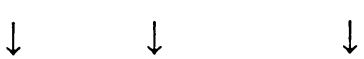


where $S / F$ is a split extension. But then by the standard theory of group extensions $S$ must be a split extension.

TheOREM 2.4. Let $\Gamma$ have property II with $\Gamma \subset S$. Then $S$ has property I.

Let $R^{s}=K \otimes R, R^{t}=F \otimes R$ and let $R^{n}$ by the direct sum of $R^{s}$ and $R^{t}$. Let $i$ denote the injection of $R^{s}$ into $R^{n}$ and $j$ the injection of $R^{t}$ into $R^{n}$. Now each $r \in R^{t}$ determines a linear automorphism of $R^{s}$. We will extend this to a linear automorphism of $R^{n}$ by assuming it acts trivially on $R^{t}$. Denote this extended automorphism by $\sigma(r)$ for $r \in R^{t}$. Then $\{\sigma(r)\}$ for all $r \in R^{t}$ is a compact subgroup of $G L(n, R)$ and hence similar to a subgroup of $O(n)$. We now assign to $r \in R^{t}$, $\sigma(r) j(r)$ and to $r^{\prime} \in R^{s}, i\left(r^{\prime}\right)$. It is then straightforward to verify that these generate a group isomorphic to $S$ in $R(n)$ having property II.

The proofs of Theorems A, B and C of the Introduction now follow easily from theorems which we have proven in $\S \S 1$ and 2.

\section{BIBLIOGRAPHY}

1. L. Auslander and M. Kuranishi, On the holonomy groups of locally euclidean spaces, Ann. of Math. vol. 65 (1957) pp. 411-415.

2. K. Iwasawa, On some types of topological groups, Ann. of Math. vol. 50 (1949) pp. 507-558.

3. A. Malcev, On a class of homogeneous spaces, Amer. Math. Soc. Translation, no. 39 (1951).

4. G. D. Mostow, Factor spaces of solvable groups, Ann. of Math. vol. 60 (1954) pp. 1-27.

5. H. C. Wang, Discrete subgroups of solvable Lie groups I, Ann. of Math. vol. 64 (1956) pp. 1-19.

Institute for Advanced Study 